\title{
Aplicação do Modelo Calgary para avaliação de familias de isodos na prática clínica*
}

\author{
The use of the Calgary Model for assessment of families of the aged in clinical practice \\ Uso de Modelo Calgary para la evaluación de familias de ancianos en la práctica clínica
}

\author{
Lúcia Silva', Regina Szylit Bousso', Sueli Aparecida Frari Galera' \\ 'Universidade de São Paulo. Escola de Enfermagem de Ribeirão Preto. \\ Centro Colaborador da Organização Mundial de Saúde para a pesquisa em enfermagem. Ribeirão Preto, SP
}

Submissão: $23 / 07 / 2008$

Aprovação: 22/06/2009

\section{RESUMO}

Este trabalho de abordagem Qualitativa tem como objetivo apresentar a experiência de avaliar famílias de idosos dependentes sob o enfoque sistêmico. Utilizou-se como referencial teórico o modelo Calgary de avaliação familiar e como estratégia metodológica o estudo de caso. Os dados foram coletados junto a cinco famílias de idosos dependentes, no período de setembro a dezembro de 2005, por meio de instrumento pré-elaborado. A aplicação deste modelo permitiu realizar a análise familiar levantando os principais aspectos de sua estrutura, desenvolvimento e funcionamento. Acredita-se que o enfermeiro deve buscar respaldo na literatura científica e adote instrumentos pertinentes, de forma a contribuir para o desenvolvimento de suas habilidades para realizar a abordagem familiar.

Descritores: Idoso; Família; Enfermagem.

\begin{abstract}
This Qualitative study has as objective to present the experience to assessment of the families of dependent aged under the systemic approach. The Calgary Model was used as theoretical framework and as methodological strategy the case study. The data had been collected with five families of dependent older person, from September of December of 2005, by means of the instrument elaborated previously. The application of this model allowed doing the familiar analysis raising the main aspects of its structure, development and functioning. We believe that the nurse must search endorsement in the scientific literature and adopts pertinent instruments to contribute for the development of its abilities to do the familiar approach.
\end{abstract}

Descriptors: Aged; Family; Nursing.

\section{RESUMEN}

Este trabajo de investigación cualitativa tiene como objetivo presentar la experiencia de evaluar familias de ancianos dependientes bajo la perspectiva sistémica. El Modelo Calgary fue utilizado como marco teórico y como estrategia metodológica el estudio de caso. Los datos fueron recogidos con cinco familias de personas mayores dependientes, en el período entre septiembre y diciembre de 2005, por medio de instrumento elaborado previamente. El uso de este modelo permitió realizar el análisis familiar a partir de los aspectos principales de su estructura, desarrollo y funcionamiento. Se cree que el enfermero debe buscar el endoso en literatura científica y adoptar los instrumentos pertinentes, para contribuir para el desarrollo de sus capacidades para realizar el abordaje familiar.

Descriptores: Anciano; Familia; Enfermería.

*Trabalho extraído de Dissertação de Mestrado apresentada à Escola de Enfermagem de Ribeirão Preto/USP, parcialmente subsidiada pelo Conselho Nacional de Desenvolvimento Científico e Tecnológico (CNPQ). 


\section{INTRODUÇÃO}

Nos países considerados desenvolvidos, instalou-se um processo de envelhecimento populacional lento ao longo dos anos, fato este Que possibilitou atenção especializada por parte das políticas públicas a esta população no decorrer de décadas ${ }^{(1)}$.

lá no Brasil, país considerado em desenvolvimento, o Instituto Brasileiro de Geografia e Estatística ${ }^{(2)}$, no levantamento censitário do ano de 2000, divulgou Que a população brasileira era de 169.799.170 habitantes e destes, 8,6\% possuíam idade igual ou superior a 60 anos e estimou Que, em 2010, o Brasil possuirá o percentual de $9,8 \%$ de idosos.

Nessa etapa da vida, ocorrem alterações funcionais Que, embora variem de um indivíduo a outro, são encontradas em todos os idosos e são próprias do processo de envelhecimento natural, acarretando, portanto, em maior predisposição do indivíduo ao surgimento de condições crônicas de saúde e suas possíveis incapacidades para o desenvolvimento de atividades, tornando o idoso dependente de cuidados.

No cenário brasileiro, a rapidez com Que vem se instalando o envelhecimento da população ${ }^{(3)}$ poderia justificar a falta de preparo da sociedade para receber o crescente número de idosos.

A literatura brasileira ${ }^{(4,5)}$ aponta para o despreparo dos serviços de saúde e dos próprios profissionais envolvidos com a saúde do idoso, o Que se configura como um obstáculo Que compromete a competência e a Qualidade da assistência prestada a essa clientela.

Em relação ao cuidado ao idoso, os profissionais devem considerar Que, além de das intervenções e do uso de tecnologias complexas, deve-se atentar também para a sobrecarga dos familiares advinda pela responsabilidade direta do cuidado, já Que esta sobrecarga pode favorecer o adoecimento do cuidador e de toda a família.

Assim, visando apreender a complexidade da experiência da velhice no âmbito familiar, deve-se compreender Que a abordagem individual centrada no idoso, em detrimento da abordagem familiar, pode conduzir a uma avaliação e conseQüente intervenção limitadas sobre as necessidades e demandas do indivíduo e da família.

Considerar a família como uma perspectiva de trabalho ainda constitui-se um desafio para os profissionais de saúde, sobretudo em um contexto em Que o cuidado está voltado, principalmente, para atender às necessidades do indivíduo e não da unidade familiar ${ }^{(6)}$.

$\mathrm{Na}$ área da geriatria e da gerontologia, ao considerar a família como um sistema, ou seja, como uma unidade na Qual é possível visualizar a interação entre seus membros, a presença de um idoso dependente de cuidados afeta todos os componentes do grupo, em graus variados, e por isso é importante Que os profissionais de saúde disponham de um instrumento de avaliação Que considere a estrutura, o desenvolvimento no decorrer do tempo e o funcionamento da família, ao proporem os cuidados a esse idoso.

A abordagem dos sistemas familiares tem sido empregada com o intuito de auxiliar na compreensão da família como unidade de cuidado e não simplesmente como a soma da individualidade de cada membro da família, em diversos contextos.

Este trabalho se constitui em um recorte de pesquisa mais extensa da área de enfermagem da família ${ }^{(7)} \mathrm{Que}$, para alcançar seu objetivo, buscou aplicar a abordagem sistêmica para a avaliação de famílias de idosos dependentes, com base no Modelo Calgary de Avaliação da Família (MCAF), uma estrutura integrada para avaliação familiar proposta por Wright e Leahey ${ }^{(8)}$.

Por se acreditar que a família como unidade de cuidado é a perspectiva que dá sentido ao processo de trabalho na Estratégia Saúde da Família(9), acredita-se ser importante divulgar esta experiência, já Que pode respaldar a atuação e ser absorvida também por outros profissionais Que atuam neste contexto.

Portanto, o objetivo deste artigo é apresentar a experiência de avaliar famílias de idosos sob a perspectiva com base no Modelo Calgary de Avaliação Familiar.

\section{REFERENCIAL TEÓRICO}

\section{Modelo Calgary de Avaliação Familiar}

Este modelo trata-se de uma estrutura multidimensional e tem sido reconhecido mundialmente e adotado em faculdades de enfermagem em países como Austrália, Grã-Bretanha, Estados Unidos, Canadá e Brasil ${ }^{(8)}$.

Baseia-se em um fundamento teórico Que envolve não somente o conceito de sistemas, mas também cibernética, comunicação e mudança, sendo constituído por três categorias principais: estrutural, de desenvolvimento e funcional, conforme ilustrado na Figura 1 .

Convém esclarecer Que é papel de cada enfermeiro selecionar as subcategorias desse Modelo a serem exploradas. Desse modo, nem todas as subcategorias são avaliadas em um primeiro encontro e algumas podem nunca ser exploradas ${ }^{(8)}$.

A categoria estrutural compreende a estrutura da família, ou seja, Quem faz parte dela, Qual é o vínculo afetivo entre seus membros em comparação com os indivíduos de fora, e Qual é o seu contexto. Três aspectos da estrutura familiar podem ser examinados prontamente: elementos internos (composição da família, gênero, orientação sexual, ordem de nascimento, subsistemas e limites), elementos externos (família extensa e sistemas mais amplos) e contexto (etnia, raça, classe social, religião e ambiente).

A categoria de desenvolvimento refere-se à transformação progressiva da história familiar durante as fases do ciclo de vida: sua história, o curso de vida, o crescimento da família, o nascimento, a morte.

Já a categoria funcional refere-se ao modo como os indivíduos da família interagem. Podem ser explorados dois aspectos: o funcionamento instrumental, Que se refere às atividades da vida cotidiana, e o funcionamento expressivo, Que diz respeito aos estilos de comunicação, solução de problemas, papéis, crenças, regras e alianças.

Acredita-se Que considerar a família do idoso dependente de cuidados como um sistema contribui para a sustentação da operacionalização de uma avaliação familiar integral, Que reconheça a sua estrutura, o desenvolvimento de suas tarefas e de seus vínculos no decorrer do ciclo de vida e o funcionamento de suas atividades e estilos de comunicação, por exemplo.

Sob essa perspectiva, a dinâmica familiar influencia a evolução do envelhecimento e da situação de dependência do indivíduo e estes, por sua vez, influenciam a dinâmica do grupo familiar, continuamente. 


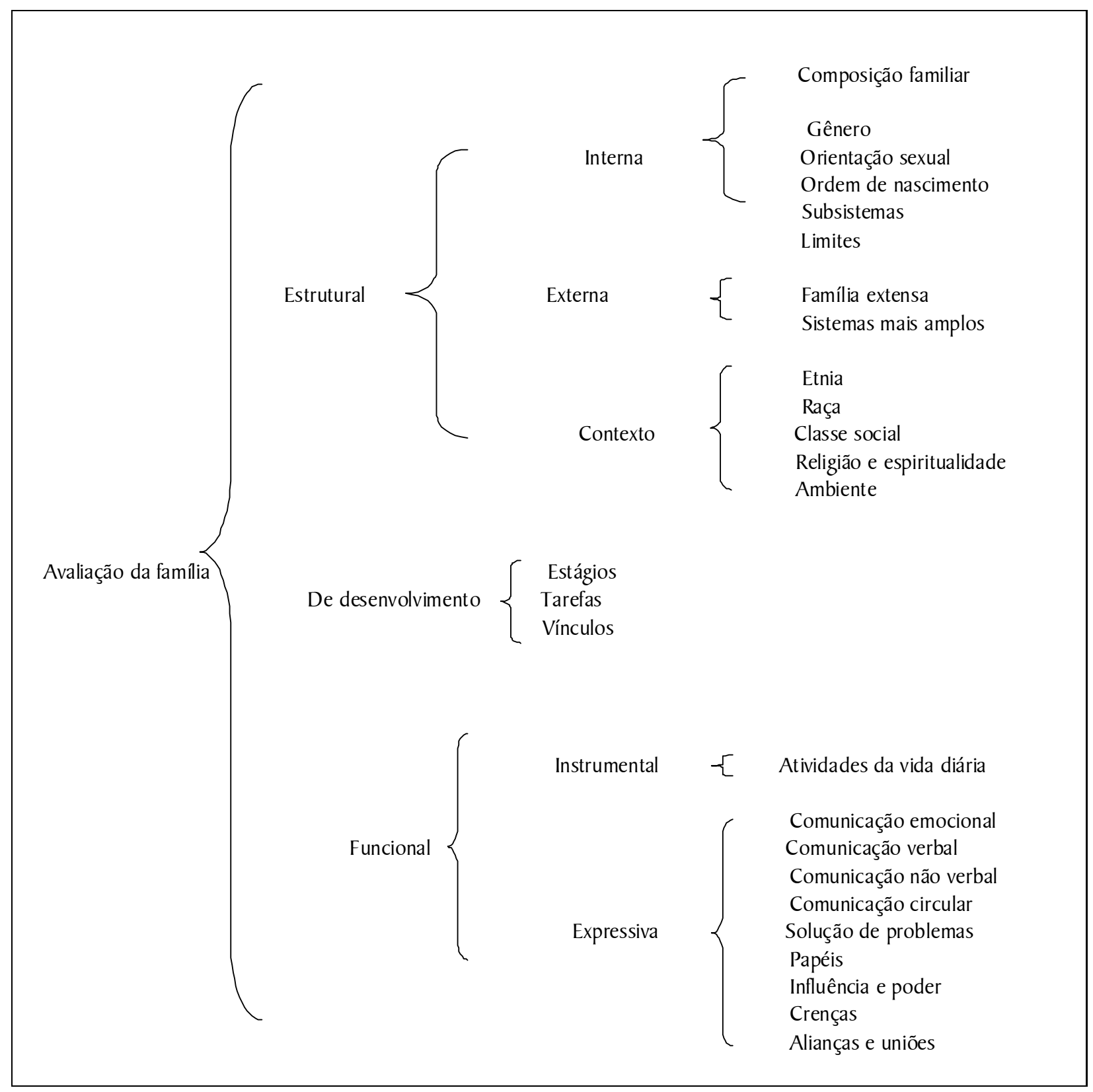

Figura 1. Diagrama ramificado do $\mathrm{MCAF}^{(8)}$.

\section{REFERENCIAL METODOLÓGICO}

Visando atingir o objetivo traçado, adotou-se como estratégia metodológica para este trabalho de abordagem Qualitativa o estudo de caso ${ }^{(10)}$, método amplo que permite ser aplicado a uma variedade de problemas e pode ser utilizado em diversas áreas de pesquisa para proporcionar maior conhecimento e envolvimento do pesQuisador com uma situação real observada.

O estudo foi realizado por meio de três encontros com cada família estudada, Que possuía um idoso dependente e oue fazia parte da área de abrangência de uma Unidade de Saúde da Família de um município do interior paulista.

Os participantes foram 11 integrantes de cinco famílias que desejaram ser incluídos na pesquisa.
Apenas as crianças ou aQueles familiares Que não tinham disponibilidade pessoal para participar dos encontros, em Qualeuer momento da realização desse trabalho, foram excluídos.

Ressalta-se Que embora nem todos os integrantes das famílias tenham participado da pesquisa, o enfoque da mesma foi a unidade familiar. Dessa forma, todos os participantes foram convidados a observar sua família, conforme preconiza o referencial sistêmico(8).

Os procedimentos da pesquisa respeitaram o constante na Declaração de Helsinki e, de acordo com a Resolução no 196 do Conselho Nacional de Saúde, de 10 de outubro de 1996, Que aprovou as diretrizes e normas regulamentadoras de pesquisas envolvendo seres humanos, os dados foram coletados mediante parecer favorável do Comitê de Ética em Pesquisa da Faculdade de Medicina de Botucatu da Universidade Estadual Paulista - UNESP 
(ofício no 452/2004) e após obtenção do consentimento livre e esclarecido dos participantes. Utilizou-se meio de instrumento préelaborado com base no Modelo Calgary de Avaliação Familiar ${ }^{(8)}$, enfocando a avaliação estrutural da família (construção do genograma, representação gráfica dos membros da família e seus relacionamentos, e ecomapa, diagrama dos relacionamentos significativos da família com outras pessoas ou com instituições), a avaliação do desenvolvimento da família ao longo do ciclo vital, com suas tarefas e vínculos e a avaliação do funcionamento instrumental (atividades da vida diária) e expressivo (estilos de comunicação, papéis, influência, crenças, alianças da família). A seguir, estes dados foram analisados à luz do próprio referencial sistêmico.

\section{RESULTADOS E DISCUSSÃO}

\section{Avaliação Estrutural}

No tocante à estrutura interna, observou-se Que, das cinco famílias participantes, apenas uma (família I) conservava a estrutura nuclear, composta pelo casal de idosos, filhos e netos. As outras Quatro (famílias 2, 3, 4 e 5) eram constituídas por apenas um dos cônjuges idosos compondo o núcleo, por motivo de viuvez do companheiro.

Em relação a isso, com a redução da família nuclear, é preciso Que o enfermeiro amplie o conceito de família de modo a abranger a família extensa (Que inclui a família de origem e a de procriação) e os sistemas mais amplos (instituições sociais mais amplas e pessoas com as Quais a família tem contato significativo), visando ampliar a rede de suporte do grupo familiar para uma assistência integral e efetiva ${ }^{(11)}$.

No tocante à estrutura externa, verificou-se Que Quatro famílias ( I , 2, 3 e 4) dispunham de diversos elementos do supra-sistema familiar, ou seja, da rede de suporte social, como igrejas, amigos e parentes.

No entanto, constatou-se Que uma família (família 5) apresentava escasso relacionamento de seus membros com os sistemas mais amplos, um dos fatos Que poderia contribuir para justificar a acentuada sobrecarga relatada pelo cuidador, visto Que a rede social constitui-se em importante forma de ajuda para o enfrentamento das dificuldades familiares do dia-a-dia ${ }^{(8)}$.

Alguns autores ${ }^{(12)}$, ao estudarem o apoio social de famílias de idosos Que residiam em área rural coberta por Unidade de Saúde da Família, observaram tendências de Que o maior número de pessoas residindo no domicílio poderia aumentar o escore de apoio material, o Que sinaliza Que domicílios multigeracionais podem constituir uma estratégia eficiente na obtenção de recursos financeiros, por exemplo.

Entretanto, é importante destacar Que nenhum membro das famílias encontrava-se desempregado até o término da fase de coleta de dados desta pesquisa.

Ademais, na realidade estudada, a Secretaria Municipal de Saúde, por meio da Estratégia Saúde da Família, supre as famílias pertencentes à área de abrangência em relação aos medicamentos de base para as condições crônicas e aos materiais para a realização de curativos e sondagem vesical. Além disso, existem recursos humanos próprios da Estratégia (médicos, enfermeiros, auxiliares de enfermagem) para prover o atendimento domiciliário (exame clínico, monitorização dos sinais vitais, realização de curativos e cuidados para manutenção da sondagem vesical, por exemplo).

Em relação ao contexto, mais especificamente ao Que diz respeito à subcategoria ambiente, verificou-se Que Quatro famílias viviam em bairros com condições sociais e sanitárias satisfatórias - famílias 2, 3 , 4 e 5 - isto é, abastecimento de água e energia elétrica e escoamento do esgoto por meio da rede pública. Somente uma família (família I) residia em um bairro caracterizado por precárias condições: algumas casas possuíam fossa ou despejavam os dejetos a céu aberto e a coleta pública do lixo não contemplava todo o bairro.

Apesar de essa família em particular (família I) não viver em um domicílio nessas condições, as áreas circunvizinhas apresentavam ambiente insalubre. Em relação a isso, é importante mencionar Que o levantamento das condições sociais e ambientais em Que vivem as famílias da área de abrangência da Estratégia Saúde da Família faz parte do papel do enfermeiro Que atua no campo da atenção primária à saúde, visto Que estas influem sobre o estado de saúde das pessoas, podendo predispor o indivíduo e a família à ocorrência de doenças.

Nesse sentido, ressalta-se que a abordagem do processo saúdedoença relaciona-se intrinsecamente ao acesso que as famílias têm às necessidades vitais, como as condições de moradia, e deve ser pautado no princípio de vigilância à saúde, ou seja, na detecção precoce de pontos críticos Que evidenciam as necessidades de saúde ${ }^{(13)}$.

Em relação à subcategoria classe social, Que também compõe a categoria contexto da avaliação estrutural, observou-se Que, diferentemente da realidade encontrada em outros estudos, Que evidenciaram baixos recursos financeiros em relação ao aumento dos gastos pelo acometimento de doenças em idosos Que convivem com a família, de uma forma geral, o rendimento mensal das famílias participantes desta pesquisa, Que variou entre 3,4 e 10 salários mínimos, pareceu ser suficiente para manter os gastos familiares. Isso poderia ser explicado pelo fato de a situação de dependência do idoso não ter implicado, necessariamente, em gastos extras para essas famílias, e sim em disponibilidade pessoal dos cuidadores para o auxílio ou supervisão dos idosos.

\section{Avaliação de desenvolvimento}

Em relação aos estágios da vida familiar, nesta pesquisa, Quatro famílias Que se encontravam no estágio $\operatorname{tardio}^{(14)}(2,3,4$ e 5) conviviam também com jovens adultos solteiros e duas famílias ( 1 e 4) conviviam com crianças peQuenas.

No planejamento da assistência Que tem como cenário um domicílio onde convivem várias gerações, como foi o caso das famílias deste estudo, o enfermeiro deve considerar Que as tarefas específicas da fase da velhice interagem com aquelas próprias das gerações dos filhos e dos netos, oferecendo respaldo para Que a própria família promova novos arranjos para o desempenho dos papéis familiares ${ }^{(14)}$.

No tocante aos vínculos, convém mencionar Que o acolhimento dos filhos observado em Quatro famílias ( 1 , 2, 3 e 4) e a dificuldade da nora no cuidado ao idoso, observado em uma família (família 5), são certamente permeados pelas Questões de gênero e pelas relações familiares. O conceito de família relacionado à idéia de consangüinidade pode fazer com Que a responsabilidade dos filhos de cuidar de seus pais seja natural, aceitável. Já no caso do cuidado prestado por uma nora, neste estudo, esta responsabilidade pareceu não ser aceita de forma tran@üila e natural, valor portanto, Que não 
poderia ser generalizado, considerando-se o tamanho da amostra.

\section{Avaliação funcional}

A avaliação instrumental permitiu constatar Que, apesar de a Questão financeira ser um fator importante para a sobrevivência familiar, neste estudo, não pareceu determinante em relação à Qualidade do cuidado dispensado ao idoso. Ao contrário: Quatro famílias (1, 2, 3 e 4), independente do rendimento familiar mensal, mantinham seus idosos em acompanhamento regular do estado de saúde, satisfatórias condições de higiene no local onde os mesmos residiam e relacionamento harmonioso entre os membros, o Que favorecia a promoção da saúde da unidade familiar.

Entretanto, na família em Que apresentava maior rendimento mensal (família 5), foram evidenciadas condições desfavoráveis para a saúde do idoso, como o precário estado de higiene do Quarto e o relacionamento familiar conflituoso, possivelmente pelo fato de a família não compreender as Questões envolvidas no processo de envelhecimento, como o agravamento da situação de dependência em decorrência de uma condição crônica.

Dos 1 l participantes desta peseuisa (sendo duas idosas dependentes), os nove familiares dos idosos constituíam os seus principais cuidadores, sendo sete mulheres e apenas dois homens.

Este dado permitiu tecer algumas considerações a respeito do funcionamento expressivo, mais especificamente em relação às crenças. A prevalência de mulheres cuidadoras é vastamente encontrada em estudos sobre o tema ${ }^{(1,15)}$, assim como também constatou-se neste trabalho; uma possível explicação para isso seria Que, culturalmente, é esperado que a mulher desempenhe o papel de cuidadora em circunstâncias Que assim o exigem, como por exemplo na ocorrência de uma condição crônica em um membro da família.
Considerar essa bagagem cultural, na prática, significa reconhecer Que este aspecto pode contribuir para o desgaste das mulheres cuidadoras da família e, nesse sentido, o enfermeiro deve empreender esforços na aQuisição de habilidades para adotar de fato a família como unidade de cuidado. Assim, a sobrecarga pode ser minimizada, desde Que o enfermeiro ajude o grupo familiar a se mobilizar no sentido de Que o maior número de familiares contribua com alguma atividade referente ao cuidado do ente idoso dependente.

\section{CONSIDERAÇÕES FINAIS}

A avaliação familiar respaldada pelo Modelo Calgary adotado neste estudo permitiu realizar a análise da família como um todo, levantando os principais aspectos de sua estrutura, desenvolvimento e funcionamento.

Sabe-se Que um dos grandes potenciais de trabalho no cenário da Estratégia Saúde da Família é a sua capacidade de oferecer suporte e de fortalecer as famílias para lidarem com situações críticas, como o envelhecimento com dependência, de forma a minimizar a sobrecarga e o sofrimento.

Entretanto, acredita-se Que apenas a determinação política Que a equipe de saúde da família deve abordar a família como unidade de cuidado é insuficiente para Que de fato isto aconteça, sendo imprescindível que o profissional busque respaldo na literatura científica e adote instrumentos pertinentes, de forma a contribuir para o desenvolvimento de suas habilidades para realizar essa abordagem.

A partir da avaliação integral da família, é possível, em parceria com seus integrantes, propor intervenções de ajuda para a melhoria da Qualidade de vida familiar, ajudando-a a vislumbrar suas próprias soluções para lidar com as dificuldades do cotidiano.

\section{REFERÊNCIAS}

1. Karsch UM. Idosos dependentes: famílias e cuidadores. Cad Saúde Pública 2003; 19(3): 86 I-6.

2. Instituto Brasileiro de Geografia e Estatística. Censo demográfico de 2000 [citado em 23 jun 2006]. Disponível em: http:// www.ibge.gov.br/home/estatistica/populacao/perfilidoso/ tabelal_2.shtm

3. Duarte YAO. O processo de envelhecimento e a assistência ao idoso. In: Ministério da Saúde (BR). Manual de enfermagem. Brasília: Ministério da Saúde; 2001. p. 185-96.

4. Diogo MID'E. Formação de recursos humanos na área de saúde do idoso. Rev Latino-am Enfermagem 2004; 12(2): 280-2.

5. Martins II, Albuquerque GL, Nascimento RP, Barra DCC, Souza WGA, Pacheco WNS. Necessidades de educação em saúde dos cuidadores de pessoas idosas no domicílio. Texto Contexto Enferm 2007; 16(2): 254-62.

6. Angelo M. O contexto familiar. In: Duarte YAO, Diogo MJD. Atendimento domiciliar: um enfoque gerontológico. São Paulo: Atheneu; 2000. p. 27-31.

7. Silva L. O processo de conviver com um idoso dependente sob a perspectiva do grupo familiar. [dissertação]. Ribeirão Preto (SP): Escola de Enfermagem de Ribeirão Preto, Universidade de São Paulo; 2007.

8. Wright LM, Leahey M. Enfermeiras e famílias: um guia para

avaliação e intervenção na família. $3^{a}$ ed. São Paulo: Roca; 2002.

9. Angelo M, Bousso RS. Fundamentos da assistência à família em saúde. In: Ministério da Saúde (BR). Manual de enfermagem. Brasília: Ministério da Saúde; 200 I. p.14-7.

10. Galdeano LE, Rossi LA, Zago MMF. Roteiro instrucional para a elaboração de um estudo de caso clínico. Rev Latino-am Enfermagem 2003; 1 I (3): 37 I-5.

I I. Carter B, McGoldrick M. As mudanças no ciclo de vida familiar: uma estrutura para a terapia familiar. In: Carter B, McGoldrick $\mathrm{M}$, organizadoras. As mudanças no ciclo de vida familiar. $2^{\mathrm{a}}$ ed. Porto Alegre: Artes Médicas; 2001. p. 7-29.

12. Pinto JLG, Garcia ACO, Bocchi SCM, Carvalhaes MABL. Características do apoio social oferecido a idosos de área rural assistida pelo PSF. Ciência Saúde Coletiva 2006; I I (3): 753-64.

13. Fracolli LA, Bertolozzi MR. A abordagem do processo saúde-doença das famílias e do coletivo. In: Ministério da Saúde (BR). Manual de Enfermagem. Brasília: Ministério da Saúde: 2001. p. 4-8.

14. Walsh F. A família no estágio tardio de vida. In: In: Carter B, McGoldrick M, organizadoras. As mudanças no ciclo de vida familiar. $2^{\mathrm{a}}$ ed. Porto Alegre: Artes Médicas; 2001. p. 269-87.

15. Silva L, Bocchi SCM. A sinalização do enfermeiro entre os papéis de familiar visitante e acompanhante de adulto e idoso. Rev Latino-am Enfermagem 2005; 13(2): 180-7. 